\title{
Clinical and biological implications of Hippo pathway dysregulation in sarcomas
}

Research Article

Anastasios Kyriazoglou1*, Roubini Zakopoulou1, Flora Zagouri,

Aristotelis Bamias ${ }^{1}$, Meletios Athanasios Dimopoulos ${ }^{1}$

${ }^{1}$ Department of Clinical Therapeutics, General Hospital Alexandra,

Athens, Greece

Received 24 September 2018; Accepted 28 February 2019

\begin{abstract}
Sarcomas are mesenchymal malignant tumors with poor prognosis and limited treatment options. Hippo pathway is a recently discovered pathway normally involved in organ development and wound healing. Hippo signaling is often altered in solid tumors. The molecular elements of Hippo signaling include MST1/2 and LATS1/2 kinases which phosphorylate and regulate the activity of YAP and TAZ COtranscriptional activators. Hippo pathway cross-talks with several molecular pathways with known oncogenic function. In sarcomas Hippo signaling plays a pivotal role in tumorigenesis, evolution and resistance in chemotherapy regimens. Targeting Hippo pathway could potentially improve prognosis and outcome of sarcoma patients.
\end{abstract}

Keywords: Sarcomas • Hippo pathway • Yap • Taz

\section{Introduction}

Sarcomas are a heterogeneous group of mesenchymal tumors. The recent WHO (World Health Organization) classification of tumors categorizes sarcomas in more than 50 entities based on their histopathological and genetic data (1). Sarcomas are malignant tumors that occur in both sexes and in almost every age of life, affecting people mainly of the $2^{\text {nd }}$ to the $6^{\text {th }}$ decade of life $(2,3)$. Treatment of sarcomas is still based on surgery and total resection of the tumor (4). Systemic treatment and/ or radiotherapy are mostly used in metastatic or high stage disease. However, to date no sarcomatargeted therapies have been approved and traditional chemotherapy, namely doxorubicin and ifosfamide remain the most effective drugs available in our armamentarium (5). The benefit from systematic chemotherapy remains poor with PFS (progression-free survival) of 5-7 months and overall survival for metastatic sarcomas of about 1113 months (6-8). The recent publication of a clinical trial phase Ib-II showed that the combination of Doxorubicin with Olaratumab - a PDGFRa inhibitor - has offered a benefit of 11.8 months in the median overall survival to the patients who received the doublet compared to those who received Doxorubicin monotherapy (9). NCCN (National Comprehensive Cancer Network) and ESMO (European Society of Medical Oncology) guidelines included this combination in their recent versions, with the ESMO experts commenting on the unknown mechanism of action of Olaratumab and the fact that the doublet was compared to Doxorubicin monotherapy and not to Doxorubicin plus Ifosfamide which is the standard of treatment in Europe $(10,11)$.

\section{Hippo Pathway elements and regulation}

Hippo signaling pathway is a developmental pathway discovered recently in Drosophila melanogaster. Hippo pathway controls organ size, tissue regeneration, wound healing and maintenance of tissue specific stem cells in mammals' development $(12,13)$. Hippo signaling pathway modulates mesenchymal stem cell fate like normal bone (osteogenic differentiation), adipocyte (adipogenic differentiation) and muscle (myogenic differentiation), which are the origins of the most common sarcomas (14). 


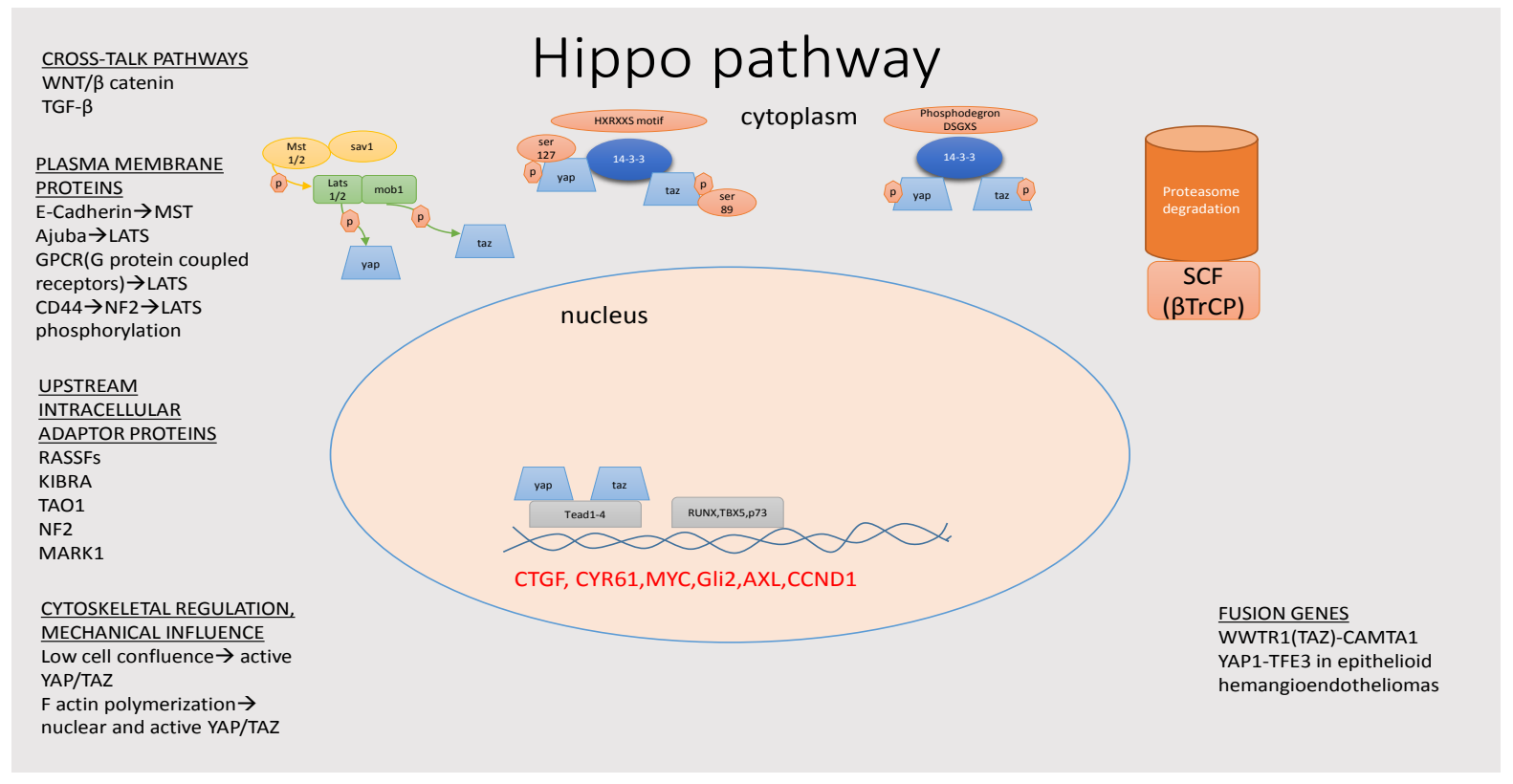

Figure 1: Hippo pathway proteins' localization and regulation.

Hippo signaling cascade includes MST1/2 and LATS1/2, two kinases which phosphorylate YAP and TAZ co-transcription factors. The phosphorylated forms of YAP and TAZ shuttle from the nucleus to the cytoplasm via their binding to 14-3-3 proteins. Ultimately, the cytoplasmic faith of pYAP and pTAZ is proteasomal degradation. The unphosphorylated forms of YAP and TAZ are rescued from 14-3-3 binding and cytoplasmic shuttling and remain in the nucleus. Thus, nuclear YAP and TAZ regulate their function through TEAD transcription factors $(15,16)$ (Figure 1).

Hippo signaling pathway is regulated through several mechanisms. Plasma membrane proteins like $\mathrm{G}$ protein coupled receptor ligands (GPCRs) have been identified as regulators of Hippo signaling (17). Upstream intracellular adaptor proteins such as NF2, RASSFs and KIBRA interact with MST1/2 and LATS1/2 alter their function and subsequently YAP and TAZ localization (18-20). Cytoskeletal remodeling is caused by mechanical changes which facilitate YAP and TAZ to emerge as important factors linking extracellular matrix signals to transcriptional outputs that regulate cell behavior $(21,22)$. Furthermore, there are many publications reporting cross-talk of Hippo with other pathways like Wnt/ b-Catenin, TGFb, PI3K, MAPK and Jak/Stat (23-26). Activation of Wnt/ b-Catenin pathway decreases Hippo pathway activity. YAP and TAZ dissociate from the proteasomal degradation complex resulting to dysregulation of Hippo signaling (23). JNK (Janus kinase pathway) cross-talks with Hippo pathway, through colocalization of AP1 (dimmer of JUN and FOS proteins) with YAP and TAZ in TEAD transcription factors $(24,27)$. Furthermore, Ajuba proteins inhibit LATS1/2 function and activate Hippo pathway (28). PI3K activation leads to Hippo pathway inhibition conferring antagonism between contact inhibition and growth promotion (29).

\section{Hippo pathway in cancer}

Recent data have shown that Hippo signaling pathway is frequently altered in several solid tumors, indicating a possible implication in their pathogenesis (12, 13). In details, comprehensive genomic analysis of Mesotheliomas has shown the presence of chromosomal translocations involving genes of the Hippo pathway as well as frequent mutations of these genes $(30,31)$. Of importance, fusion transcripts of Hippo pathway lead to inactivation of their tumor suppressor function promoting carcinogenesis (30). Also in Mesotheliomas strong dysregulation of Hippo pathway increases the transcription levels of cell cycle promoting genes like CyclinD1 (32). YAP and TAZ in Hepatocellular carcinoma contribute to disease progression by conferring stem cell-like properties (33). In Gastric cancer the downregulation of MST1/2 and LATS1/2 result to YAP,TAZ nuclear localization and Hippo pathway activation (34). YAP overexpression is related to shorter overall survival and TNM stage 
in human Colorectal cancer patients (34). In Oral Squamus cell carcinoma YAP and TAZ are shown to drive protumorigenic signals (35). Hippo pathway is also engaged in Breast cancer, promoting several functions such as epithelial to mesenchymal transition, stem cell generation and therapeutic resistance (36).

\section{Hippo pathway in sarcomas}

Deregulation of Hippo pathway is also implicated in pathogenesis of sarcomas. This was initially demonstrated in preclinical models. Transgenic Hippo mouse models frequently develop sarcomas (37-39). Mouse models developing Rhabdomyosarcomas reveal the importance of YAP in this fatal sarcoma type (40).

In clinical samples, translocations with Hippo pathway genes have been detected in various sarcomas. In epithelioid hemangioendotheliomas YAP and TAZ create fusion genes resulting in YAP-TFE3 and TAZ-CAMTA1 respectively $(41,42)$. Clear cell sarcoma of the kidney and endometrial stromal sarcomas are characterized by the presence of the fusion gene YWHAE-FAM22. This fusion gene creates a nuclear fusion protein with a 14-3-3 element which interacts with YAP and TAZ $(43,44)$. Copy number alterations of YAP and TAZ have been reported in sarcomas, though mutations of these genes are not very common (45-47). Epigenetic regulation of Hippo elements have been shown in Ewing sarcomas and Osteosarcomas (48-50). FOXM1 interacts with YAP and promotes cell proliferation and tumorigenesis in a subset of soft tissue sarcomas (47). Despite all this descriptive evidence, a mechanistic biological understanding of Hippo pathway involvement in sarcomas is remarkably lacking.

In Rhabdomyosarcoma the characteristic PAXFOX01 fusion oncogene interacts with Hippo pathway to drive tumor development (40). Embryonal Rhabdomyosarcomas (eRMS) present with Ras activating mutations, while YAP is upregulated in eRMS. In Ras mutated RMS cell lines and in murine xenografts YAP promotes cell proliferation, decreases apoptosis and disrupts myogenic differentiation. Pharmacologic depletion of YAP with verteporfin decreased cell growth, showing that this molecular lesion is an early step in RMS tumorigenesis (51). TAZ, the second effector of Hippo pathway acts as an oncogene in eRMS. Its expression is correlated to Myf5 upregulation, an eRMS stem cell factor. TAZ is associated with poor survival in eRMS (52).

YAP is stabilized in Ewing sarcoma through the interaction of BMI-1 (53). In this sarcoma type YAP is expressed in tumor samples tested, however there is not a clear connection of survival and YAP expression. On the other hand, Akt expression in the same tumor samples was statistically related to survival, conferring a possible interaction of Hippo with Akt signaling (54).

In Osteosarcoma (OS) tumor samples YAP/TAZ and b-integrin were associated with prognosis. YAP and TAZ were independent prognostic factors for PFS (progression free survival) (55). Experiments looking for the molecular targets of Hippo signaling in Osteosarcoma revealed TEAD1 transcription factor to be the main Hippo effector. Cyr61 and PTGS2 were the downstream targets overexpressed in osteosarcoma cell lines (56). TAZ overexpression is accompanied by miR-224 overexpression, a TAZ phenocopy which inhibits tumor suppressor SMAD4, thus facilitating proliferation and migration of OS cells (57).

\section{Hippo pathway and Drugs}

Hippo pathway deregulation has been implicated in resistance to chemotherapeutic drugs (58). Overexpression of YAP and TAZ in BRAF V600E mutant melanoma cells confers resistance to BRAF inhibitors (59). In BRAF V600E mutant lung cells YAP expression correlates with resistance to MEK and RAF inhibitors (60). TAZ is shown to mediate resistance to taxol in Breast cancer cells (61).

Hippo pathway can be used as a target for therapeutic intervention. Small molecules like Verteporfin, statins and biphosphonates are being evaluated as putative treatment options $(62,63)$. There are few published reports of drug studies targeting Hippo pathway in sarcoma cells (64). Clinical trials of Phase I, II and III with drugs inhibiting Hippo pathway are still on-going (65-67).

In osteosarcoma cell lines YAP was reported to participate in chemoresistance. MG63 cells overexpressing YAP showed accelerated proliferation compared to YAP knocked out cells and presented resistance to high concentrates of chemotherapeutic drugs such as methotrexate and doxorubicin (68).

\section{Conclusions and future challenges}

Since Hippo signaling pathway constitutes a recently identified pathway, there are yet many critical aspects understudied. The oncogenic function of Hippo pathway is a "hot spot" of current cancer research efforts. Hippo pathway plays a crucial role in sarcoma stem cell formation, proliferation and resistance to chemotherapy regimens. Hippo pathway cross-talks with several other 
molecular pathways and confers new insights in the complexity of the molecular cascades of sarcomas' tumorigenesis and evolution. Targeting Hippo pathway in sarcomas, where there is a great need of targeted therapies, could potentially improve prognosis and outcome in patients suffering from these lethal tumors.

\section{References}

1] Fletcher $C D$. The evolving classification of soft tissue tumours - an update based on the new 2013 WHO classification. Histopathology. 2014;64(1):2-11.

[2] Gatta G, van der Zwan JM, Casali PG, Siesling S, Dei Tos AP, Kunkler I, et al. Rare cancers are not so rare: the rare cancer burden in Europe. Eur J Cancer. 2011;47(17):2493-511.

[3] Gustafson P. Soft tissue sarcoma. Epidemiology and prognosis in 508 patients. Acta Orthop Scand Suppl. 1994;259:1-31.

[4] Gronchi A, Lo Vullo S, Colombo C, Collini P, Stacchiotti S, Mariani L, et al. Extremity soft tissue sarcoma in a series of patients treated at a single institution: local control directly impacts survival. Ann Surg. 2010;251(3):506-11.

[5] Sleijfer S, Ray-Coquard I, Papai Z, Le Cesne A, Scurr M, Schoffski P, et al. Pazopanib, a multikinase angiogenesis inhibitor, in patients with relapsed or refractory advanced soft tissue sarcoma: a phase II study from the European organisation for research and treatment of cancer-soft tissue and bone sarcoma group (EORTC study 62043). J Clin Oncol. 2009;27(19):3126-32.

[6] Ratan R, Patel SR. Chemotherapy for soft tissue sarcoma. Cancer. 2016.

[7] Gordon EM, Sankhala KK, Chawla N, Chawla SP. Trabectedin for Soft Tissue Sarcoma: Current Status and Future Perspectives. Adv Ther. 2016;33(7):1055-71.

[8] Schoffski P, Chawla S, Maki RG, Italiano A, Gelderblom H, Choy E, et al. Eribulin versus dacarbazine in previously treated patients with advanced liposarcoma or leiomyosarcoma: a randomised, open-label, multicentre, phase 3 trial. Lancet. 2016;387(10028):1629-37.

[9] Tap WD, Jones RL, Van Tine BA, Chmielowski $B$, Elias AD, Adkins D, et al. Olaratumab and doxorubicin versus doxorubicin alone for treatment of soft-tissue sarcoma: an open-label phase $1 \mathrm{~b}$ and randomised phase 2 trial. Lancet. 2016;388(10043):488-97.

[10] Casali PG, Abecassis N, Bauer S, Biagini R, Bielack S, Bonvalot S, et al. Soft tissue and visceral sarcomas: ESMO-EURACAN Clinical Practice Guidelines for diagnosis, treatment and follow-up. Ann Oncol. 2018;29(Supplement_4):iv51-iv67.

[11] von Mehren M, Randall RL, Benjamin RS, Boles S, Bui MM, Ganjoo KN, et al. Soft Tissue Sarcoma, Version 2.2018, NCCN Clinical Practice Guidelines in Oncology. J Natl Compr Canc Netw. 2018;16(5):536-63.

[12] Varelas $X$. The Hippo pathway effectors TAZ and YAP in development, homeostasis and disease. Development. 2014;141(8):1614-26.

[13] Zhang K, Qi HX, Hu ZM, Chang YN, Shi ZM, Han $\mathrm{XH}$, et al. YAP and TAZ Take Center Stage in Cancer. Biochemistry. 2015;54(43):6555-66.

[14] Mo JS, Park HW, Guan KL. The Hippo signaling pathway in stem cell biology and cancer. EMBO Rep. 2014;15(6):642-56.

[15] Ehmer U, Sage J. Control of Proliferation and Cancer Growth by the Hippo Signaling Pathway. Mol Cancer Res. 2016;14(2):127-40.

[16] Stein C, Bardet AF, Roma G, Bergling S, Clay I, Ruchti A, et al. YAP1 Exerts Its Transcriptional Control via TEAD-Mediated Activation of Enhancers. PLoS Genet. 2015;11(8):e1005465.

[17] Yu FX, Zhao B, Panupinthu N, Jewell JL, Lian I, Wang LH, et al. Regulation of the Hippo-YAP pathway by G-protein-coupled receptor signaling. Cell. 2012;150(4):780-91.

[18] Avruch J, Praskova M, Ortiz-Vega S, Liu M, Zhang XF. Nore1 and RASSF1 regulation of cell proliferation and of the MST1/2 kinases. Methods Enzymol. 2006;407:290-310.

[19] Yu J, Zheng Y, Dong J, Klusza S, Deng WM, Pan D. Kibra functions as a tumor suppressor protein that regulates Hippo signaling in conjunction with Merlin and Expanded. Dev Cell. 2010;18(2):288-99.

[20] Kim S, Jho EH. Merlin, a regulator of Hippo signaling, regulates Wnt/beta-catenin signaling. BMB Rep. 2016;49(7):357-8.

[21] Halder G, Dupont S, Piccolo S. Transduction of mechanical and cytoskeletal cues by YAP and TAZ. Nat Rev Mol Cell Biol. 2012;13(9):591-600. 
[22] Rauskolb C, Sun S, Sun G, Pan Y, Irvine KD Cytoskeletal tension inhibits Hippo signaling through an Ajuba-Warts complex. Cell. 2014;158(1):14356.

[23] Piersma B, Bank RA, Boersema M. Signaling in Fibrosis: TGF-beta, WNT, and YAP/TAZ Converge. Front Med (Lausanne). 2015;2:59.

[24] Zanconato F, Forcato M, Battilana G, Azzolin L, Quaranta E, Bodega B, et al. Genome-wide association between YAP/TAZ/TEAD and AP-1 at enhancers drives oncogenic growth. Nat Cell Biol. 2015;17(9):1218-27.

[25] Lin L, Bivona TG. The Hippo effector YAP regulates the response of cancer cells to MAPK pathway inhibitors. Molecular \& Cellular Oncology. 2016;3(1):e1021441.

[26] Fan R, Kim N-G, Gumbiner BM. Regulation of Hippo pathway by mitogenic growth factors via phosphoinositide 3-kinase and phosphoinositidedependent kinase-1. Proceedings of the National Academy of Sciences. 2013;110(7):2569-74.

[27] Liu X, Li H, Rajurkar M, Li Q, Cotton JL, Ou J, et al. Tead and AP1 Coordinate Transcription and Motility. Cell Rep. 2016;14(5):1169-80.

[28] Sun G, Irvine KD. Ajuba family proteins link JNK to Hippo signaling. Sci Signal. 2013;6(292):ra81.

[29] Huang W, Lv X, Liu C, Zha Z, Zhang $H$, Jiang $\mathrm{Y}$, et al. The $\mathrm{N}$-terminal phosphodegron targets TAZ/WWTR1 protein for SCFbetaTrCP-dependent degradation in response to phosphatidylinositol 3-kinase inhibition. J Biol Chem. 2012;287(31):26245-53.

[30] Miyanaga A, Masuda M, Tsuta K, Kawasaki K, Nakamura Y, Sakuma T, et al. Hippo Pathway Gene Mutations in Malignant Mesothelioma: Revealed by RNA and Targeted Exon Sequencing. Journal of Thoracic Oncology.10(5):844-51.

[31] Bueno R, Stawiski EW, Goldstein LD, Durinck S, De Rienzo A, Modrusan Z, et al. Comprehensive genomic analysis of malignant pleural mesothelioma identifies recurrent mutations, gene fusions and splicing alterations. Nat Genet. 2016;48(4):407-16.

[32] Mizuno $T$, Murakami $H$, Fujii $M$, Ishiguro $F$, Tanaka I, Kondo Y, et al. YAP induces malignant mesothelioma cell proliferation by upregulating transcription of cell cycle-promoting genes. Oncogene. 2012;31(49):5117-22.

[33] Hayashi H, Higashi T, Yokoyama N, Kaida T, Sakamoto K, Fukushima Y, et al. An Imbalance in TAZ and YAP Expression in Hepatocellular Carcinoma Confers Cancer Stem Cell-like Behaviors Contributing to Disease Progression. Cancer Res. 2015;75(22):4985-97.
[34] Kang W, Cheng AS, Yu J, To KF. Emerging role of Hippo pathway in gastric and other gastrointestinal cancers. World J Gastroenterol. 2016;22(3):1279-88.

[35] Hiemer SE, Zhang L, Kartha VK, Packer TS, Almershed M, Noonan V, et al. A YAP/TAZRegulated Molecular Signature Is Associated with Oral Squamous Cell Carcinoma. Mol Cancer Res. 2015;13(6):957-68.

[36] Maugeri-Sacca M, Barba M, Pizzuti L, Vici P, Di Lauro L, Dattilo R, et al. The Hippo transducers TAZ and YAP in breast cancer: oncogenic activities and clinical implications. Expert Rev Mol Med. 2015;17:e14.

[37] Yang Z, Zhang M, Xu K, Liu L, Hou WK, Cai YZ, et al. Knockdown of YAP1 inhibits the proliferation of osteosarcoma cells in vitro and in vivo. Oncol Rep. 2014;32(3):1265-72.

[38] Giovannini M, Robanus-Maandag E, van der Valk M, Niwa-Kawakita M, Abramowski V, Goutebroze L, et al. Conditional biallelic Nf2 mutation in the mouse promotes manifestations of human neurofibromatosis type 2. Genes Dev. 2000;14(13):1617-30.

[39] Nishio M, Hamada K, Kawahara K, Sasaki M, Noguchi F, Chiba S, et al. Cancer susceptibility and embryonic lethality in Mob1a/1b double-mutant mice. J Clin Invest. 2012;122(12):4505-18.

[40] Crose LE, Galindo KA, Kephart JG, Chen C, Fitamant $\mathrm{J}$, Bardeesy $\mathrm{N}$, et al. Alveolar rhabdomyosarcomaassociated PAX3-FOX01 promotes tumorigenesis via Hippo pathway suppression. J Clin Invest. 2014;124(1):285-96.

[41] Tanas MR, Ma S, Jadaan FO, Ng CK, Weigelt B, Reis-Filho JS, et al. Mechanism of action of a WWTR1(TAZ)-CAMTA1 fusion oncoprotein. Oncogene. 2016;35(7):929-38.

[42] Antonescu CR, Le Loarer F, Mosquera JM, Sboner A, Zhang L, Chen CL, et al. Novel YAP1-TFE3 fusion defines a distinct subset of epithelioid hemangioendothelioma. Genes Chromosomes Cancer. 2013;52(8):775-84.

[43] O'Meara E, Stack D, Lee CH, Garvin AJ, Morris T, Argani $\mathrm{P}$, et al. Characterization of the chromosomal translocation $\mathrm{t}(10 ; 17)(\mathrm{q} 22 ; \mathrm{p} 13)$ in clear cell sarcoma of kidney. J Pathol. 2012;227(1):72-80.

[44] Lee $\mathrm{CH}$, Ou WB, Marino-Enriquez A, Zhu M, Mayeda M, Wang $Y$, et al. 14-3-3 fusion oncogenes in high-grade endometrial stromal sarcoma. Proc Natl Acad Sci U S A. 2012;109(3):929-34.

[45] Mohamed AD, Tremblay AM, Murray GI, Wackerhage $\mathrm{H}$. The Hippo signal transduction pathway in soft tissue sarcomas. Biochim Biophys Acta. 2015;1856(1):121-9. 
[46] Deel MD, Li JJ, Crose LE, Linardic CM. A Review: Molecular Aberrations within Hippo Signaling in Bone and Soft-Tissue Sarcomas. Front Oncol. 2015;5:190.

[47] Eisinger-Mathason TS, Mucaj V, Biju KM, Nakazawa MS, Gohil M, Cash TP, et al. Deregulation of the Hippo pathway in soft-tissue sarcoma promotes FOXM1 expression and tumorigenesis. Proc Natl Acad Sci U S A. 2015;112(26):E3402-11.

[48] Gharanei S, Brini AT, Vaiyapuri S, Alholle A, Dallol $A$, Arrigoni $E$, et al. RASSF2 methylation is a strong prognostic marker in younger age patients with Ewing sarcoma. Epigenetics. 2013;8(9):893-8.

[49] Avigad S, Shukla S, Naumov I, Cohen IJ, Ash S, Meller I, et al. Aberrant methylation and reduced expression of RASSF1A in Ewing sarcoma. Pediatr Blood Cancer. 2009;53(6):1023-8.

[50] Richter AM, Walesch SK, Wurl P, Taubert H, Dammann RH. The tumor suppressor RASSF10 is upregulated upon contact inhibition and frequently epigenetically silenced in cancer. Oncogenesis. 2012;1:e18.

[51] Slemmons KK, Crose LE, Rudzinski E, Bentley $\mathrm{RC}$, Linardic CM. Role of the YAP Oncoprotein in Priming Ras-Driven Rhabdomyosarcoma. PLoS One. 2015;10(10):e0140781.

[52] Mohamed A, Sun C, De Mello V, Selfe J, Missiaglia E, Shipley J, et al. The Hippo effector TAZ (WWTR1) transforms myoblasts and TAZ abundance is associated with reduced survival in embryonal rhabdomyosarcoma. J Pathol. 2016;240(1):3-14.

[53] Hsu JH, Lawlor ER. BMI-1 suppresses contact inhibition and stabilizes YAP in Ewing sarcoma. Oncogene. 2011;30(17):2077-85.

[54] Ahmed AA, Abedalthagafi M, Anwar AE, Bui MM. Akt and Hippo Pathways in Ewing's Sarcoma Tumors and Their Prognostic Significance. J Cancer. 2015;6(10):1005-10.

[55] Bouvier C, Macagno N, Nguyen Q, Loundou A, Jiguet-Jiglaire C, Gentet JC, et al. Prognostic value of the Hippo pathway transcriptional coactivators YAP/TAZ and beta1-integrin in conventional osteosarcoma. Oncotarget. 2016;7(40):64702-10.

[56] Chai J, Xu S, Guo F. TEAD1 mediates the oncogenic activities of Hippo-YAP1 signaling in osteosarcoma. Biochem Biophys Res Commun. 2017;488(2):297302.

[57] Ma J, Huang K, Ma Y, Zhou M, Fan S. The TAZmiR-224-SMAD4 axis promotes tumorigenesis in osteosarcoma. Cell Death Dis. 2017;8(1):e2539.
[58] Cordenonsi M, Zanconato F, Azzolin L, Forcato M, Rosato A, Frasson C, et al. The Hippo transducer TAZ confers cancer stem cell-related traits on breast cancer cells. Cell. 2011;147(4):759-72.

[59] Kim MH, Kim J, Hong $\mathrm{H}$, Lee $\mathrm{SH}$, Lee JK, Jung $\mathrm{E}$, et al. Actin remodeling confers BRAF inhibitor resistance to melanoma cells through YAP/TAZ activation. EMBO J. 2016;35(5):462-78.

[60] Lin L, Sabnis AJ, Chan E, Olivas V, Cade L, Pazarentzos E, et al. The Hippo effector YAP promotes resistance to RAF- and MEK-targeted cancer therapies. Nat Genet. 2015;47(3):250-6.

[61] Lai D, Ho KC, Hao Y, Yang X. Taxol resistance in breast cancer cells is mediated by the hippo pathway component TAZ and its downstream transcriptional targets Cyr61 and CTGF. Cancer Res. 2011;71(7):2728-38.

[62] Park HW, Guan KL. Regulation of the Hippo pathway and implications for anticancer drug development. Trends Pharmacol Sci. 2013;34(10):581-9.

[63] Ye S, Eisinger-Mathason TS. Targeting the Hippo pathway: Clinical implications and therapeutics. Pharmacol Res. 2016;103:270-8.

[64] Bhat UG, Halasi M, Gartel AL. FoxM1 is a general target for proteasome inhibitors. PLoS One. 2009;4(8):e6593.

[65] Huggett MT, Jermyn M, Gillams A, Illing R, Mosse $\mathrm{S}$, Novelli $\mathrm{M}$, et al. Phase I/II study of verteporfin photodynamic therapy in locally advanced pancreatic cancer. Br J Cancer. 2014;110(7):1698704.

[66] Chan KK, Oza AM, Siu LL. The statins as anticancer agents. Clin Cancer Res. 2003;9(1):10-9.

[67] Fenske TS, Shah NM, Kim KM, Saha S, Zhang $C$, Baim AE, et al. A phase 2 study of weekly temsirolimus and bortezomib for relapsed or refractory B-cell non-Hodgkin lymphoma: A Wisconsin Oncology Network study. Cancer. 2015;121(19):3465-71.

[68] Wang DY, Wu YN, Huang JQ, Wang W, Xu M, Jia JP, et al. Hippo/YAP signaling pathway is involved in osteosarcoma chemoresistance. Chin J Cancer. 2016;35:47. 